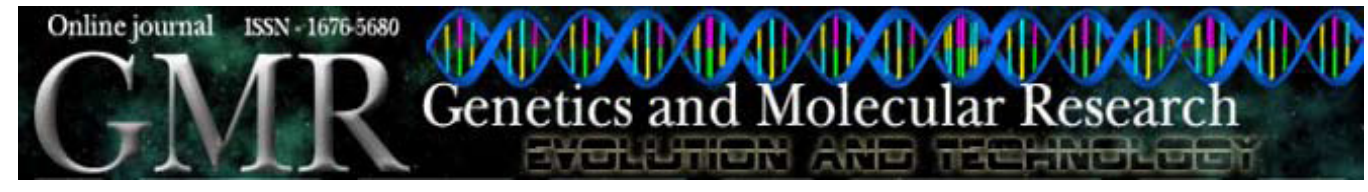

\title{
Microdissection testicular sperm extraction causes spermatogenic alterations in the contralateral testis
}

\author{
A.B. Oliveira Filho ${ }^{1}$, R.S. Souza ${ }^{2}$, M.T.V. Azeredo-Oliveira ${ }^{2}$, \\ R.L. Peruquetti ${ }^{2}$ and A.P. Cedenho ${ }^{1}$ \\ ${ }^{1}$ Departamento de Cirurgia, Divisão de Urologia, \\ Seção de Reprodução Humana, Universidade Federal de São Paulo, \\ São Paulo, SP, Brasil \\ ${ }^{2}$ Departamento de Biologia, Instituto de Biociências, \\ Letras e Ciências Exatas de São José do Rio Preto, \\ Universidade Estadual Paulista, São José do Rio Preto, SP, Brasil \\ Corresponding author: A.B. Oliveira Filho \\ E-mail: uroandrologia@gmail.com
}

Genet. Mol. Res. 9 (3): 1405-1413 (2010)

Received March 24, 2010

Accepted May 20, 2010

Published July 20, 2010

DOI 10.4238/vol9-3gmr860

\begin{abstract}
Testicular sperm extraction (TESE) associated with intracytoplasmic sperm injection has allowed many men presenting non-obstructive azoospermia to achieve fatherhood. Microdissection TESE (microTESE) was proposed as a method to improve sperm retrieval rates in these patients; however, there have been failures. Little is known about whether microTESE leads to spermatogenic alterations in the contralateral testis. We assessed histological outcomes of experimental microTESE in the contralateral testis of adult male rabbits. Nine adult male rabbits were divided into three groups: control (testicular biopsy to observe normal histological and morphometric values), sham (incision of the tunica vaginalis, and a contralateral testicular biopsy to observe histological and morphometric patterns, 45 days later), and study (left testicular microTESE, and a right testicular biopsy to observe
\end{abstract}


histological and morphometric patterns, 45 days later). Sections were assessed by calculating Johnsen-like scores, and measuring total tubule diameter, lumen diameter and epithelial height. The results were compared using ANOVA and Bonferroni's statistical analysis. Morphometric evaluation of the seminiferous tubules did not demonstrate differences between the three groups. However, microTESE caused spermatogenic alterations, leading to maturation arrest in the contralateral testis.

Key words: Testis biopsy; Microdissection; Seminiferous tubules; Surgery

\section{INTRODUCTION}

Testicular surgical techniques for sperm retrieval, such as testicular sperm extraction (TESE) and microdissection testicular sperm extraction (M-TESE), are prerequisites for treating infertile men with non-obstructive azoospermia (Ramasamy et al., 2005). Azoospermia is prevalent in $2 \%$ of infertile men. Non-obstructive azoospermia due to spermatogenic failure may be classified as primary or secondary. Primary cases may be of genetic origin, such as in Y-chromosome microdeletions, or of congenital origin as in cryptorchidism, for example (Gordon, 2002). Secondary causes may be due to chemo- or radiotherapy or endocrine dysfunctions (Anniballo et al., 2000). Testicular histology generally demonstrates maturation arrest or complete absence of germ cells (Cedenho et al., 2004). Testicular failure in these men may coexist with a remaining parenchyma that still maintains an immeasurable spermatogenic capacity. Thus, spermatogenic potential may vary from areas showing complete spermatogenesis to tubular sclerosis (Shulze et al., 1999).

Intracytoplasmic sperm injection (ICSI) has allowed these men to achieve fatherhood after TESE. TESE has been used therapeutically since 1995 with ICSI, both in obstructive azoospermia patients, if microepididymal sperm aspiration renders negative results, and in non-obstructive azoospermia patients (Ramasamy et al., 2005). On the other hand, M-TESE has been increasingly used due to better results in obtaining testicular sperm, when compared to TESE (Devroey et al., 1995; Schlegel and Su, 1997; Schlegel, 1999). Failure to retrieve sperm in non-obstructive azoospermia patients may be as high as $57 \%$ of patients (Friedler et al., 1997; Rosenlund et al., 1998; Okada et al., 2002).

However, although M-TESE has appeared as a technical advancement, a few concerns have been raised, such as: i) what are the possible negative effects on the contralateral testis; ii) how should testicular intervention through M-TESE be planned, and iii) should only one testis be approached or both testes simultaneously?

In order to answer these questions, the present study set out to investigate testicular spermatogenic activity in adult rabbits following M-TESE.

\section{MATERIAL AND METHODS}

Nine adult (7-8 months old) male New Zealand rabbits with an average weight of 3100 $\mathrm{g}$ were included in the study. The animals were divided into three groups: control, sham, and study group. Institutional Review Board approval was obtained from the São Paulo Federal 
University Research Ethics Committee, and guidelines established by the National Guide for the Care and Use of Laboratory Animals (National Research Council, 1996) were followed.

The animals were deprived of food and water for a 12-h period prior to the surgical procedures. Blood samples were collected for analyses (hematocrit, hemoglobin, and leukocytes), and the animals were subsequently weighed and subjected to anesthesia using Ketalar ${ }^{\circledR}$ + Coopazine ${ }^{\circledR}$ (1.0 to $1.5 \mathrm{~mL}$ intramuscular). Antisepsis was performed using topical povidine.

\section{Control group}

Left testicular biopsy was performed and three distinct fragments of testicular parenchyma were excised by standard procedures. The tissue was diced into smaller fragments and immersed in Bouin's solution. The testis fragments were embedded in paraffin. Sections (1-3 $\mu \mathrm{m}$ thick) were obtained and prepared using hematoxylin-eosin (HE) staining. After biopsy, the animals were euthanized by respiratory arrest using a high anesthetic dose.

\section{Sham group}

The sham group was initially submitted only to surgical incision of the tunica vaginalis in the left testis, but preserving the tunica albuginea. After 45 days, right testicular biopsy was performed, and three distinct fragments of testicular parenchyma were excised, diced into smaller fragments, and immersed in Bouin's solution. The testis fragments were embedded in paraffin. Sections (1-3 $\mu \mathrm{m}$ thick) were obtained and prepared using HE staining. After biopsy, the animals were euthanized by respiratory arrest using a high anesthetic dose.

\section{Study group}

In this group, the animals were submitted to M-TESE using all phases of surgical technique: a) exposure of the left testis by a $1.0-\mathrm{cm}$ incision made in the subcutaneous cellular tissue using a JENA-OPS-341 surgical microscope (Carl Zeiss Inc., Thornwood, Germany) under $8 \mathrm{X}$ magnification; b) a 1.0-cm longitudinal incision was made in the tunica albuginea at the median region of the lateral portion of the testis, parallel to the larger caliber sub-capsular vessel; c) testicular parenchyma microfragments were removed and discarded; d) the tunica albuginea and the skin were sutured using synthetic Prolene ${ }^{\circledR} 7-0$. The testis fragments were embedded in paraffin. Sections (1-3 $\mu \mathrm{m}$ thick) were obtained and prepared using HE staining. After 45 days, right testicular biopsy was performed and the animals were euthanized by respiratory arrest using a high anesthetic dose.

\section{Histological analyses}

Samples from the three groups were examined under light microscopy using an Axioskop 2 microscope (Carl Zeiss Inc.); the images were analyzed using Image Pro Plus (Media Cybernetics, Silver Spring, USA) and Axio Vision 3.1 (Carl Zeiss Inc.). For analysis of spermatogenesis and morphometry, only fields containing cross-sections of seminiferous tubules were captured, using 200X magnification. The Johnsen score (Johnsen, 1970) is the 
usual tool to classify human spermatogenesis, and it was adapted to be used in the rabbit spermatogenesis analysis. The adapted Johnsen score was called the Johnsen-like score. This Johnsen-like score was used to assess rabbit spermatogenesis (Table 1). Morphometric parameters assessed were: total tubule diameter (TD, $\mu \mathrm{m})$, epithelial height $(\mathrm{EH}, \mu \mathrm{m})$, and lumen diameter $(\mathrm{LD}, \mu \mathrm{m})$.

\begin{tabular}{ll} 
Table 1. Johnsen-like scores for evaluation of rabbit spermatogenesis adapted from Johnsen $(1970)$. \\
\hline Score & Description \\
\hline 10 & Complete spermatogenesis with mature sperm cells \\
9 & Some sperm cells, with a disorganized epithelium \\
8 & Presence of few sperm $(<5$ to 10$)$ \\
7 & No sperm cells, presence of spermatids \\
6 & No sperm cells, few spermatids $(<5$ to 10$)$ \\
5 & No sperm cells or spermatids, presence of spermatocytes \\
4 & No sperm cells or spermatids, few spermatocytes $(<5)$ \\
3 & Only spermatogonia present \\
2 & Sertoli cells only \\
1 & No cells visualized in the tubular cross-section \\
\hline
\end{tabular}

\section{Statistical analysis}

Groups were compared using one-way analysis of variance (ANOVA) and when differences were found, a Bonferroni's multiple comparison post hoc test was performed to determine individual differences. An $\alpha$ value of 0.05 was used for statistical significance in this study.

\section{RESULTS}

\section{Blood analysis and body weight}

No differences were observed in blood variables (hematocrit, hemoglobin, and leukocytes) or in body weight between the three groups, nor in blood parameters before the first surgery and after the 45-day period (sham and study groups), with both groups showing only a slight increase in body weight.

\section{Spermatogenesis evaluation}

Spermatogenesis was assessed using the Johnsen-like score, as well as morphometric analysis (TD, LD, EH) of the seminiferous tubules.

Figure 1A, B, C, D, E, and F correspond to cross-sections of the seminiferous tubules of control animals indicating respective Johnsen-like scores of 10, 9, 8, 7, 6, and 5 . Standardization of Johnsen-like scores is represented in Table 2. Scores of 6 and 5 were more frequently found in the study group than in both control and sham groups, while the score 8 was less frequent in the study group than in the other two groups. No cross-sections showed a Johnsen-like score of less than 5. 

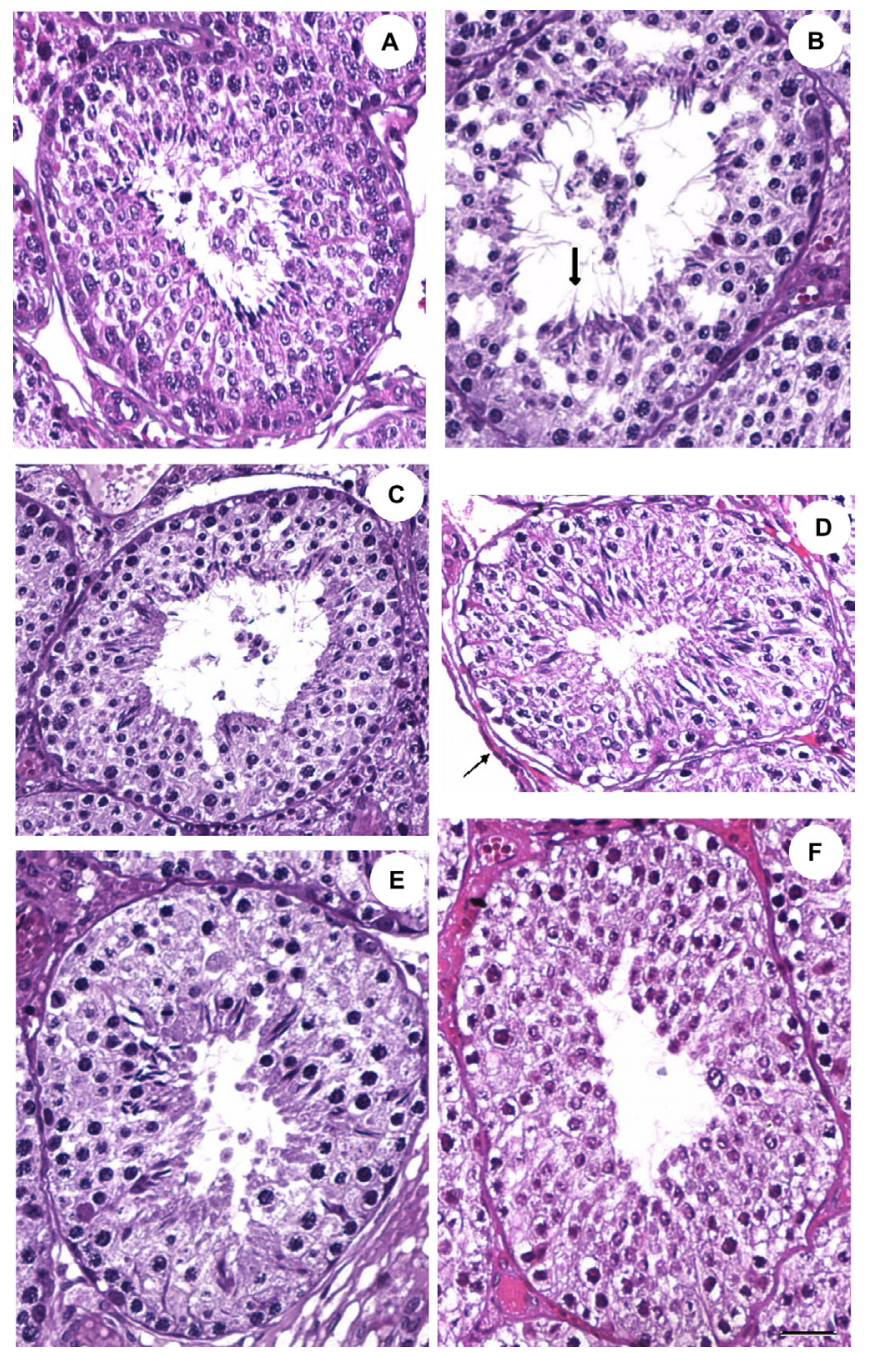

Figure 1. Standardization of Johnsen-like scores in the seminiferous tubule cross-sections of control group rabbits stained with hematoxylin-eosin (HE). A. Johnsen-like score 10. B. Johnsen-like score 9. C. Johnsen-like score 8. D. Johnsen-like score 7. E. Johnsen-like score 6. F. Johnsen-like score 5. Thin arrow $=$ basal lamina; thick arrow $=$ spermatozoon. Bar $=20 \mu \mathrm{m}$.

Table 2. Frequency and percentage of Johnsen-like scores 10, 9, 8, 7, 6, and 5 in multiple seminiferous tubule cross-sections in control, sham and study groups.

\begin{tabular}{|c|c|c|c|c|c|c|c|}
\hline \multirow[t]{2}{*}{ Group } & \multirow[t]{2}{*}{ Number of sections } & \multicolumn{6}{|c|}{ Scores } \\
\hline & & 10 & 9 & 8 & 7 & 6 & 5 \\
\hline Control & 99 & $33(34 \%)$ & $15(15 \%)$ & $5(5 \%)$ & $21(21 \%)$ & $8(8 \%)$ & $14(14 \%)$ \\
\hline Sham & 64 & $25(39 \%)$ & $20(31 \%)$ & $15(23 \%)$ & $15(23 \%)$ & $4(6 \%)$ & $5(8 \%)$ \\
\hline \multirow[t]{2}{*}{ Study } & 104 & $35(34 \%)$ & $22(21 \%)$ & $1(1 \%)$ & $20(19 \%)$ & $13(13 \%)$ & $22(21 \%)$ \\
\hline & & $\mathrm{P}>0.05$ & $\mathrm{P}>0.05$ & $\mathrm{P}<0.01 *$ & $\mathrm{P}>0.05$ & $\mathrm{P}<0.001^{*}$ & $\mathrm{P}<0.005^{*}$ \\
\hline
\end{tabular}

*Statistically significant difference $(\mathrm{P}<0.05)$. 


\section{Morphometric evaluation of the seminiferous tubule}

The TD, LD and EH of control, sham and study group animals were measured. The results for these morphometric evaluations of seminiferous tubules are displayed in Table 3. No differences were observed between the three groups.

\begin{tabular}{lcccc}
\multicolumn{5}{l}{ Table 3. Tubule and lumen diameters and epithelium height in control, sham, and study groups. } \\
\hline Group & Control & Sham & Study & P \\
\hline Number of sections & 99 & 64 & 104 & - \\
Tubule diameter & $194.3 \pm 4.5$ & $169.8 \pm 2.5$ & $188.5 \pm 6.9$ & $>0.05$ \\
Epithelium height & $59.9 \pm 1.5$ & $5.7 \pm 2.5$ & $6.3 \pm 1.7$ & $>0.05$ \\
Lumen diameter & $74.5 \pm 3.0$ & $60.4 \pm 4.0$ & $65.9 \pm 5.8$ & $>0.05$ \\
\hline
\end{tabular}

Data are reported as mens $\pm \mathrm{SD}$ in $\mu \mathrm{m}$.

\section{DISCUSSION}

The 1990's represented a major breakthrough in human reproductive medicine on account of the development of gamete micromanipulation techniques for in vitro fertilization and sperm retrieval methods for both obstructive and non-obstructive azoospermic individuals.

The most widely used technique for sperm retrieval in men with testicular dysfunction - TESE - shows overall failure rates that may exceed 57\% (Rosenlund et al., 1998; Schlegel, 1999). In order to improve the success rate of sperm retrieval, Schlegel (1999) proposed a new technique - M-TESE - in which the use of a surgical microscope allowed the inspection of focal tubular engorgement areas and demonstrated higher retrieval rates.

On the other hand, the use of M-TESE has raised uncertainties as to whether the technique may cause lesions to blood vessels within the tunica albuginea, and thus lead to histological alterations of the contralateral testis.

$\mathrm{FSH}, \mathrm{LH}$ and testosterone levels are not good parameters to access the presence of sperm in azoospermic patients (Steinberger et al., 1974; Afzelius and Mossberg, 1988; Ezeh et al., 1999). Moreover, non-obstructive azoospermic patients exhibit different histological patterns (Shulze et al., 1999). In this respect, deciding on which is the better of two techniques has posed a dilemma in reproductive medical treatments, since better does not necessarily mean less innocuous.

The present study, therefore, set out to assess possible histological alterations in the contralateral testis of New Zealand rabbits 45 days after M-TESE, was performed in the same manner as in humans.

In the present study, spermatogenesis evaluation consisted of quantifying the frequency of the different Johnsen-like scores in many sections in each group. The scores $10,9,8$, 7,6 , and 5 were observed with different frequencies within the same histological sample or between different animals. Scores of 4, 3, 2, or 1 were not observed in any sample.

The study group showed a higher frequency of scores 6 and 5 and a lower frequency of score 8 when compared to control and sham groups. Scores of 6 and 5 are representative of maturation arrest or incomplete spermatogenesis, which means that in this group, the seminiferous epithelium in the contralateral testis was damaged after M-TESE. This group also dis- 
played quantitative cytological alterations characterized by the prevalence of spermatogonia and spermatids associated with a histological pattern of maturation arrest.

FSH and testosterone are essential hormones for spermatogenesis during puberty and in adulthood. FSH receptor is localized in Sertoli cells, whereas androgen receptor is localized in Sertoli cells, Leydig cells and peritubular cells, but not in germ cells. Hence, hormonal support for germ cells is mediated by Sertoli cells (Sharpe, 1994). Although the important role of FSH and testosterone is well known, the mechanisms of hormone action on target cells are still unclear. Recent studies involving knockout models of androgen receptor (De Gendt et al., 2004) and FSH or FSH receptor (Johnston et al., 2004) as well as hypogonadal mice (Haywood et al., 2003) elucidated the particular role for each hormone. Testosterone can qualitatively support normal spermatogenesis, whereas quantitatively normal spermatogenesis requires both FSH and testosterone. FSH alone can support spermatogonial proliferation, whereas meiotic and postmeiotic differentiation requires testosterone action synergistic with FSH.

Some authors suggest that violation of the blood-testis barrier and the section of subcapsular blood vessels in the tunica albuginea may expose sperm antigens to the bloodstream, thus leading to the production of an autoimmune response. The resulting inflammatory response may alter both Leydig and Sertoli cell function, and thus affect spermatogenesis (Witkin, 1988; Osegbe, 1991; Watanabe et al., 1992; Chen and Schlegel, 2002).

In view of our results demonstrating a difference in testicular histology between rabbits submitted to M-TESE and control rabbits, along with the associated literature data regarding testosterone and androgen receptors in Leydig, Sertoli and peritubular myoid cells, it may be suggested that M-TESE causes intraparenchymatous arteriolar lesions, which may explain the alterations in spermatogenic regulatory mechanisms.

It has been previously shown that TESE leads to a decrease in blood plasma testosterone levels for up to three months, with a gradual recovery of $85 \%$ in 12 months and $90 \%$ in 18 months. The authors also demonstrated that the number of TESE procedures also impacts testosterone levels (Chen and Schlegel, 2002).

In the present study, we suggest that in these animals rupture of the blood-testis barrier during M-TESE leads to local inflammatory reaction and ultimately to the production of an auto-immune response, which alters spermatogenesis in the contralateral testis. Osegbe (1991) demonstrated - in patients with unilateral orchiepididymitis and a large number of infiltrated mononuclear and polymorphonuclear cells, and hematoma - the same inflammatory pattern in the contralateral testis associated with a decrease in the germ cell population.

Furthermore, Schlegel and Su (1997) suggested that testicular surgery, through inflammatory response, may lead to spermatogenic arrest. This was also demonstrated in the present study, in which the study group showed a higher frequency of Johnsen-like scores of 6 and 5 for cross-sections - compatible with maturation arrest - than did sham and control groups.

When comparing values for morphometric analyses among the three groups, no differences were observed in TD, LD or EH. It is likely that proliferation of spermatogonia is responsible for maintaining epithelial height in a compensatory response after M-TESE. These results are similar to those observed after TESE. Jezek et al. (1998) demonstrated the maintenance of epithelial height, and established a correlation among the presence of mature spermatids, normal tubule diameter and the presence of sperm.

Schlegel and $\mathrm{Su}$ (1997) found that, following TESE, areas of hematoma, fibrosis, and loss of vascularization are observed within the testes, and therefore, a 6-month interval be- 
tween procedures should be observed to minimize these effects. It is known that normal men produce from 80 to 100 million sperm per day, while men with non-obstructive azoospermia produce very few sperm (Shulze et al., 1999; Gordon, 2002). The present study was carried out in adult male rabbits that were healthy and fertile, and taking into account that M-TESE is performed only in human testes with testicular dysfunction, the effects observed in this study would be intensified in patients undergoing this procedure.

Finally, the results of the present study allow us to conclude that M-TESE causes spermatogenic alterations leading to maturation arrest in the contralateral testis, even if the morphometric values in the seminiferous tubules remain unaltered. Concerning humans, the new surgical M-TESE technique should be carefully considered before application.

\section{REFERENCES}

Afzelius BA and Mossberg GB (1988). The Immobile-Cilia Syndrome Including Kartagner's Syndrome. In: The Metabolic Basis of Inherited Disease (Fawcett DW, Wyngarden JB and Fredrickson DS, eds.). McGraw-Hill, New York, 2739-2750.

Anniballo R, Ubaldi F, Cobellis L, Sorrentino M, et al. (2000). Criteria predicting the absence of spermatozoa in the Sertoli cell-only syndrome can be used to improve success rates of sperm retrieval. Hum. Reprod. 15: 2269-2277.

Cedenho AP, Da Ros CT and Juliano RV (2004). Treatment of non-obstructive azoospermia. Int. Braz. J. Urol. 29 (Suppl 5): 39-41.

Chen DY and Schlegel PN (2002). Change in serum testosterone after testicular sperm extraction. J. Urol. $167: 309$.

De Gendt K, Swinnen JV, Saunders PT, Schoonjans L, et al. (2004). A Sertoli cell-selective knockout of the androgen receptor causes spermatogenic arrest in meiosis. Proc. Natl. Acad. Sci. U. S. A. 101: 1327-1332.

Devroey P, Liu J, Nagy Z, Goossens A, et al. (1995). Pregnancies after testicular sperm extraction and intracytoplasmic sperm injection in non-obstructive azoospermia. Hum. Reprod. 10: 1457-1460.

Ezeh UI, Taub NA, Moore HD and Cooke ID (1999). Establishment of predictive variables associated with testicular sperm retrieval in men with non-obstructive azoospermia. Hum. Reprod. 14: 1005-1012.

Friedler S, Raziel A, Strassburger D, Soffer Y, et al. (1997). Testicular sperm retrieval by percutaneous fine needle sperm aspiration compared with testicular sperm extraction by open biopsy in men with non-obstructive azoospermia. Hum. Reprod. 12: 1488-1493.

Gordon UD (2002). Assisted conception in the azoospermic male. Hum. Fertil. 5: S9-S14.

Haywood M, Spaliviero J, Jimemez M, King NJ, et al. (2003). Sertoli and germ cell development in hypogonadal (hpg) mice expressing transgenic follicle-stimulating hormone alone or in combination with testosterone. Endocrinology 144: 509-517.

Jezek D, Knuth UA and Schulze W (1998). Successful testicular sperm extraction (TESE) in spite of high serum follicle stimulating hormone and azoospermia: correlation between testicular morphology, TESE results, semen analysis and serum hormone values in 103 infertile men. Hum. Reprod. 13: 1230-1234.

Johnsen SG (1970). Testicular biopsy score count - a method for registration of spermatogenesis in human testes: normal values and results in 335 hypogonadal males. Hormones 1: 2-25.

Johnston H, Baker PJ, Abel M, Charlton HM, et al. (2004). Regulation of Sertoli cell number and activity by folliclestimulating hormone and androgen during postnatal development in the mouse. Endocrinology 145: 318-329.

National Research Council (1996). Guide for the Care and Use of Laboratory Animals. National Academy Press, Washington.

Okada H, Dobashi M, Yamazaki T, Hara I, et al. (2002). Conventional versus microdissection testicular sperm extraction for nonobstructive azoospermia. J. Urol. 168: 1063-1067.

Osegbe DN (1991). Testicular function after unilateral bacterial epididymo-orchitis. Eur. Urol. 19: 204-208.

Ramasamy R, Yagan N and Schlegel PN (2005). Structural and functional changes to the testis after conventional versus microdissection testicular sperm extraction. Urology 65: 1190-1194.

Rosenlund B, Kvist U, Ploen L, Rozell BL, et al. (1998). A comparison between open and percutaneous needle biopsies in men with azoospermia. Hum. Reprod. 13: 1266-1271.

Schlegel PN (1999). Testicular sperm extraction: microdissection improves sperm yield with minimal tissue excision. Hum. Reprod. 14: 131-135. 
Schlegel PN and Su LM (1997). Physiological consequences of testicular sperm extraction. Hum. Reprod. 12: 1688-1692. Schulze W, Thoms F and Knuth UA (1999). Testicular sperm extraction: comprehensive analysis with simultaneously performed histology in 1418 biopsies from 766 subfertile men. Hum. Reprod. 14 (Suppl 1): 82-96.

Sharpe RM (1994). Regulation of Spermatogenesis. In: The Physiology of Reproduction (Knobil E and Neill JD, eds.). Raven Press, New York, 1363-1433.

Steinberger E, Steinberger A and Sanborn B (1974). Endocrine control of spermatogenesis. Basic Life Sci. 4: 163-181.

Watanabe D, Sawada K, Koshimizu U, Kagawa T, et al. (1992). Characterization of male meiotic germ cell-specific antigen (Meg 1) by monoclonal antibody TRA 369 in mice. Mol. Reprod. Dev. 33: 307-312.

Witkin SS (1988). Mechanisms of active suppression of the immune response to spermatozoa. Am. J. Reprod. Immunol. Microbiol. 17: 61-64. 